\title{
Pulsed power L-band magnetron with increased operating parameters
}

Emil Szkop, Michał Rychlewski, Dariusz Baczewski, Martyna Woźniak, Andrzej Różycki, et al.

Emil Szkop, Michał Rychlewski, Dariusz Baczewski, Martyna Woźniak, Andrzej Różycki, Paweł Majewski, Mariusz Blazejewicz, Dariusz Laskowski, "Pulsed power L-band magnetron with increased operating parameters," Proc. SPIE 11442, Radioelectronic Systems Conference 2019, 1144208 (11 February 2020); doi: 10.1117/12.2565256

SPIE. Event: Radioelectronic Systems Conference 2019, 2019, Jachranka, Poland 


\title{
PULSED POWER L-BAND MAGNETRON WITH INCREASED OPERATING PARAMETERS.
}

\author{
Emil SZKOP ${ }^{1}$, Michał RYCHLEWSKI ${ }^{1}$, Dariusz BACZEWSKI ${ }^{1}$, Martyna WOŹNIAK ${ }^{1}$, Andrzej \\ RÓŻYCKI $^{1}$, Paweł MAJEWSKI ${ }^{1}$, Mariusz BŁAŻEJEWICZ ${ }^{1}$, Dariusz LASKOWSKI ${ }^{2}$ \\ [1] R\&D Department KUBARA LAMINA S.A. ul. Puławska 34, 05-500 Piaseczno, Poland \\ [2] Military University of Technology, ul. gen. Sylwestra Kaliskiego 2, 00-908 Warsaw, Poland
}

\begin{abstract}
The megawatt pulsed power magnetron operating in the L-band was designed based on magnetrons produced in the past at Kubara Lamina. Unlike its predecessors, it is characterized by much higher operating parameters: supply voltage and anode current, which translates into an increase in the obtained output power. The magnetron was designed using software for numerical simulations of the interaction of an electric charge with an alternating electromagnetic field (Particle in Cell - PiC). Aspects such as microwave matching of individual components of the device and thermal resistance of the entire system, including the appropriate type of cooling, have also been subjected to numerical analysis. In order to obtain the proper parameters of power supply of the tube, a dedicated impulse modulator powered by supercapacitors was build. The modifications allowed to obtain a power signal with a sharp and stable edge and a pulse length of the length of single microseconds. Obtaining the optimal power source had a key impact on the tube's operation. The measurements of tube output power in the waveguide system with dummy load were carried out, during which the output power of the megawatts range was obtained. This magnetron is a tube with the highest output power among the microwave tubes ever designed in the Kubara Lamina Company and probably throughout Poland.
\end{abstract}

Keywords: magnetron, CST, MAGIC, simulations, microwave tubes

\section{INTRODUCTION}

Most of the magnetrons produced today, used for both commercial and military purposes, are based on constructions developed many years ago. These are proven structures that for many years met the needs of this type of equipment, and their parameters were sufficient for the target applications. Today, despite access to specialized software for modeling physical phenomena, as well as the increasing computing power of computers, designing a new type of magnetron is still not a trivial task, and refining the structure takes many months. Although specialized programs make modeling the operation of microwave tubes with crossed fields possible, which significantly facilitates and speeds up the design process of magnetrons, practice shows that there are many factors that cannot be predicted or correctly reflected in computer models, and which have a significant impact on the operation of the device. After making a prototype of the device, before performing tests in real conditions of tube operation, it is necessary to, among others, match the power signal with adequate stability and repeatability, seasoning of the tube for many hours, or analyzing the behavior of the tube with a different operating mode than the basic mode, which may occur in real conditions, e.g. during the rise of the power pulse [10]. KUBARA LAMINA S.A. undertook the design, development and implementation of a high-power magnetron operating in the $\mathrm{L}$ band. The technological facilities, knowledge of microwave tubes and modern numerical simulation software allowed to create a new magnetron prototype and conduct tests that highlighted not only problems that arise during this kind of project, but also ways to solve them.

\section{BASICS OF MAGNETRON OPERATION}

Magnetron is a tube generating waves in the microwave frequency range, which bases its operation on the phenomenon of exciting electromagnetic oscillations in a constant magnetic field [4] [7] [10]. In the interaction area between the cylindrical cathode and the anode, under the applied a constant voltage, energy is transferred from the cloud of electrons emitted from the cathode to the oscillating high-frequency field formed in the anode resonance system. In the interaction area of the magnetron, in the presence of a magnetic field oriented parallel to the cathode axis, the electron cloud rotates

Radioelectronic Systems Conference 2019, edited by Piotr Kaniewski, Jan Matuszewski, Proc. of SPIE Vol. 11442, $1144208 \cdot$ @ 2020 SPIE · CCC code: 0277-786X/20/\$21 · doi: 10.1117/12.2565256 
around the cathode. With the right supply voltage and magnetic field induction, it is possible to achieve synchronism between the rotating electrons formed in the shape of spokes and the alternating electromagnetic field, as a result of which electron energy is given back into the high frequency field. The anode block has an even number of resonator cavities, which may have various shapes, depending on the parameters and purpose of the magnetron [2]. The solution often used in classic magnetrons are the so-called compensation rings that connect every other anode segment with each other. This ensures adequate separation of the desired mode of oscillations from other, undesirable types of higher order modes. By modifying the supply voltage value and introducing small changes in the interaction area, one can affect the type of oscillations generated. Magnetron is equipped with an output circuit that receives RF energy from the resonance system and transfers it to the waveguide [5].

\section{DESIGNING MAGNETRON}

\subsection{Design process}

In the first step, analytical calculations of the designed magnetron need to be carried out. They allow the initial determination of the geometrical dimensions of the resonance structure and estimation of the required operating parameters. After creating a computer model, it is necessary to carry out numerical Eigenmode simulations to find the resonance frequencies of the structure, and, most importantly, the separation of the basic mode from the other modes. Analytical calculations do not always find all spurious modes that may occur around the basic mode. The next step is to run a full simulation of the working tube with space charge, taking into account the interaction between crossed electric and magnetic fields, including the interaction of charged particles [3]. The PiC (Particle-In-Cell) method for electromagnetic calculations is only available in the few commercially available programs [11]. Numerical simulations allow optimization of critical geometrical dimensions of the structure to achieve the best possible output power and device efficiency at the designed operating parameters. Experience in working with simulation software shows that if a tube works properly in a PiC simulation, it will also work in reality with similar power conditions [9].

\subsection{Target design of High Power Magnetron}

Due to the requirements for high stability of oscillations generated by the designed pulsed power magnetron, a design based on a magnetron with specific Q-factor was chosen. This magnetron has an anode with 8 resonance slotted-cylindrical cavities, and double compensation rings on both sides. The parameters related to the frequency and stability of operation for calculating the magnetron's construction parameters were adopted:

- Wavelength of basic operating mode: $\lambda_{\pi}$,

- Pulling figure: $\Delta f_{p}=5 \mathrm{MHz}[10]$,

- The separation between the basic mode and the adjacent spurious mode (mode $N / 2-1)$ is: $\gamma_{\alpha} \approx 35 \%$.

In order to obtain the designed operating frequency $f_{r}$, one needs to carry out complete calculations of the resonance structure. The initial dimensions of the interaction area, cathode, and anode block with specific resonant frequency were designed from scratch, based on similar magnetrons produced in the past in the Company.

The source of electrons in the designed magnetron is a tungsten cathode impregnated with an emission paste, which has feature of using both thermal emission and secondary emission due to reverse bombardment. Magnetron has a power output circuit in the form of a double loop, which, at the end is coupled with a rectangular waveguide.

An additional goal of the design process was to design the magnetron configuration that meet the assumptions not only in relation to the maximum power value, but also that can function in the widest possible range of power. For this purpose, an analysis of the course of variation in the characteristics of threshold voltages was carried out, which showed that the adjustment of the range of oscillations power values generated in the magnetron is possible by determining the operating point using the appropriate value of the magnetic field induction intensity in the impact area. The condition for the excitation of magnetron oscillations is the synchronization of the rotation speed of electron spokes around the interaction area with the phase velocity of one of the spatial harmonics propagating in the resonant structure. The line $U(B H)$ defines the theoretical threshold of oscillation excitation with specific modes, which is given by the formula:

$$
\begin{gathered}
B=\frac{2 r_{a}}{r_{a}^{2}-r_{k}^{2}}\left(\frac{2 m U^{2}}{e}\right)^{\frac{1}{2}}\left[1+\frac{1}{2} \frac{e U_{H}}{m c^{2}}\right]^{\frac{1}{2}} \\
U_{B H}=B \frac{r_{a}^{2}-r_{k}^{2}}{2 r_{a}} v_{f}-\frac{m c^{2}}{e}\left\{1-\left[1-\left(\frac{v_{f}}{c}\right)^{2}\right]^{\frac{1}{2}}\right\}
\end{gathered}
$$


where: $v_{f}=\frac{2 \pi f_{n} r_{a}}{\gamma}, \gamma=n+p N, f_{n}$ - frequency of $n$ mode oscillations, $\gamma$ - order of oscillations corresponding to the number of electron spokes, $n$ - type of oscillations, $p$ - order of spatial harmonic, $N$ - number of resonators.

Figure 1 shows the Hull parabola and Buneman-Hartree threshold voltage characteristics for the basic mode $\pi$ and the spurious $\pi-1$ of the designed magnetron [8].

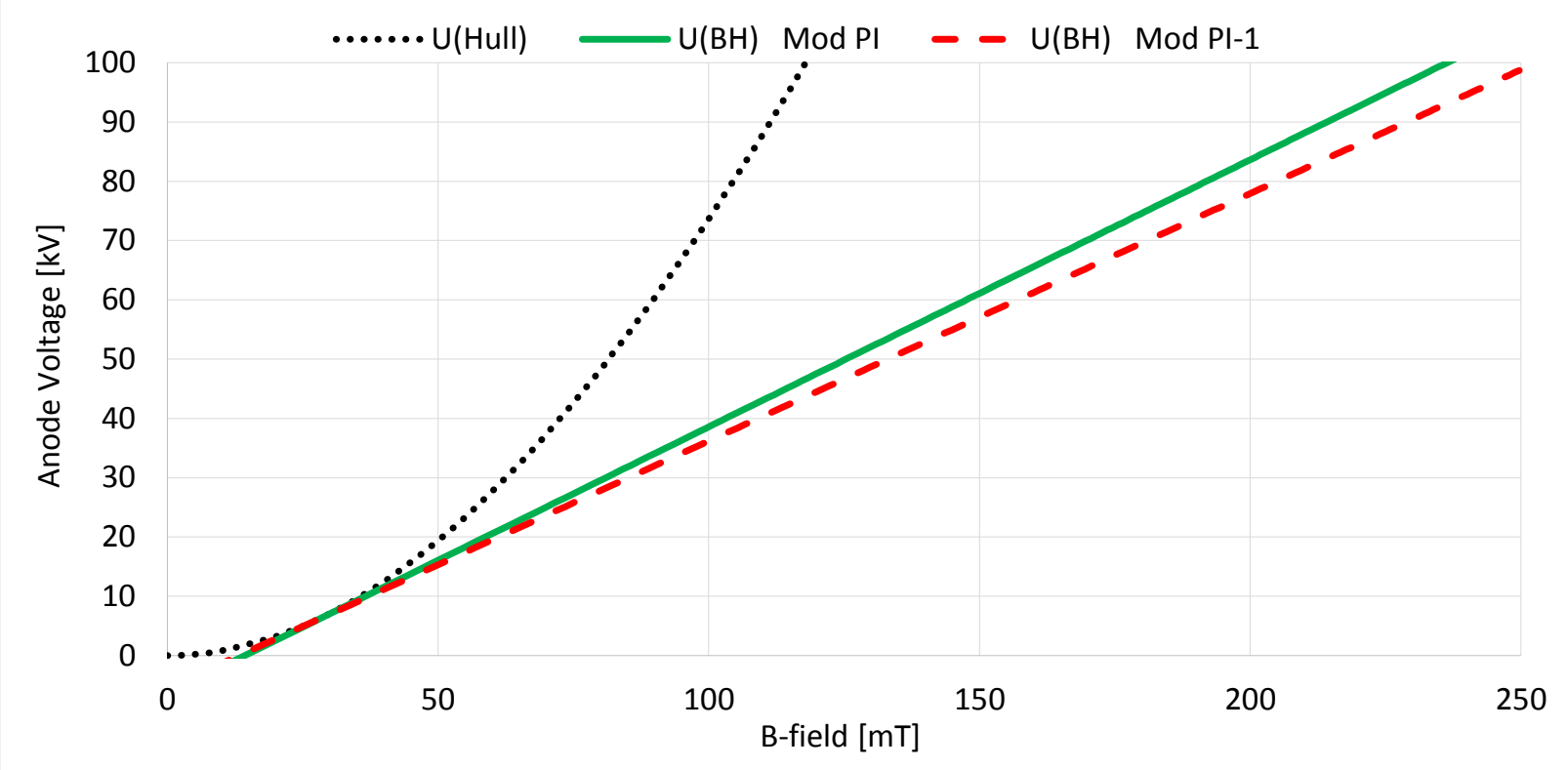

Figure 1.Buneman - Hartree conditions for designed magnetron

Optimal parameters of the magnetic field and anode voltage that will give the most stable operation with the $\pi$ mode should be above the line $U(B H)$ for the $\pi$ mode.

\section{SIMULATION RESULTS}

After conducting preliminary analytical calculations of the designed lamp geometry, the designed model was verified using the Simulia CST Studio software. First were basic Eigenmode simulations can find resonance frequencies of a given structure. The difference of the values obtained from analytical calculations and resonance frequencies obtained in Eigenmode simulations for the $\pi$ mode and adjacent spurious modes was less than $1 \%$. In the next step, minor modifications were made to the model of tube and it was adapted to simulate the full operation of the device. The following additions were introduced in the simulation: power source, power output and definition of cathode emission area. At this stage, by analyzing the preliminary results, it was possible to find certain factors that excluded a given structure from further use, such as the lack of excitation of oscillations or too strong competitiveness of parasitic modes for the environment of a given operating parameters. Formation of the electron spokes could also be observed (Figure 2.). If for a given geometry the tube was working in $\pi$ mode under the assumed conditions, one could proceed to further simulations aimed at finding optimal operating parameters, rise time of input signal, anode voltage and B-field, and optimizing output power and device efficiency. As a result of the analysis, the concept of the resonance structure was changed, finally choosing the version with 6 resonance cavities.

The analysis was conducted for three different values of supply voltage: $60 \mathrm{kV}, 80 \mathrm{kV}$ and $100 \mathrm{kV}$. The magnetic field induction value was changed for the adopted supply voltage values. The results in the form of diagrams of the dependence of the output power on the magnetic field induction value are presented in Figure 3. 


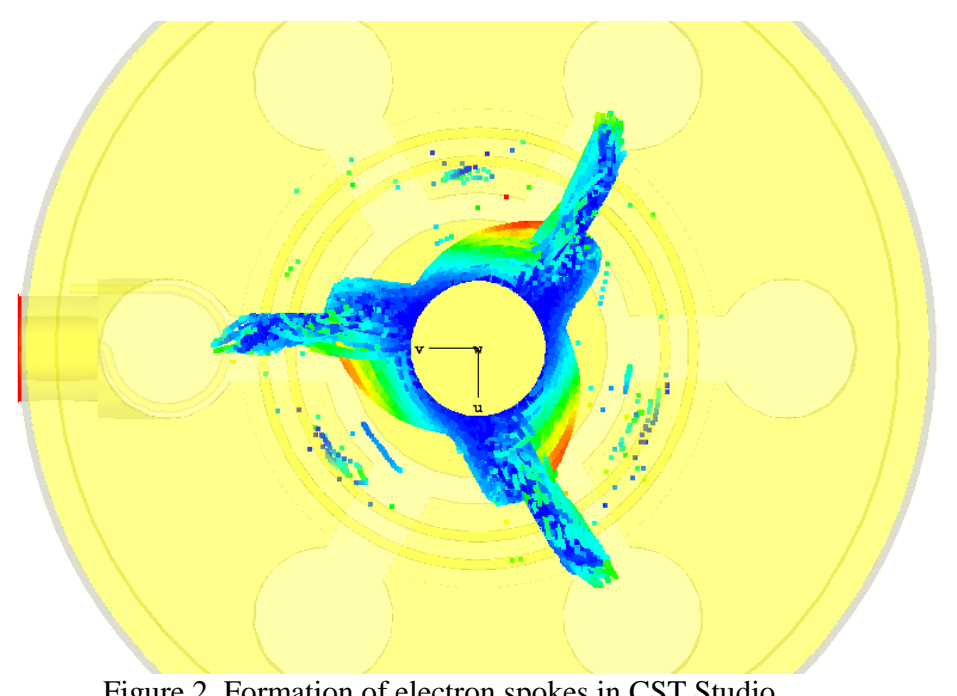

Figure 2. Formation of electron spokes in CST Studio

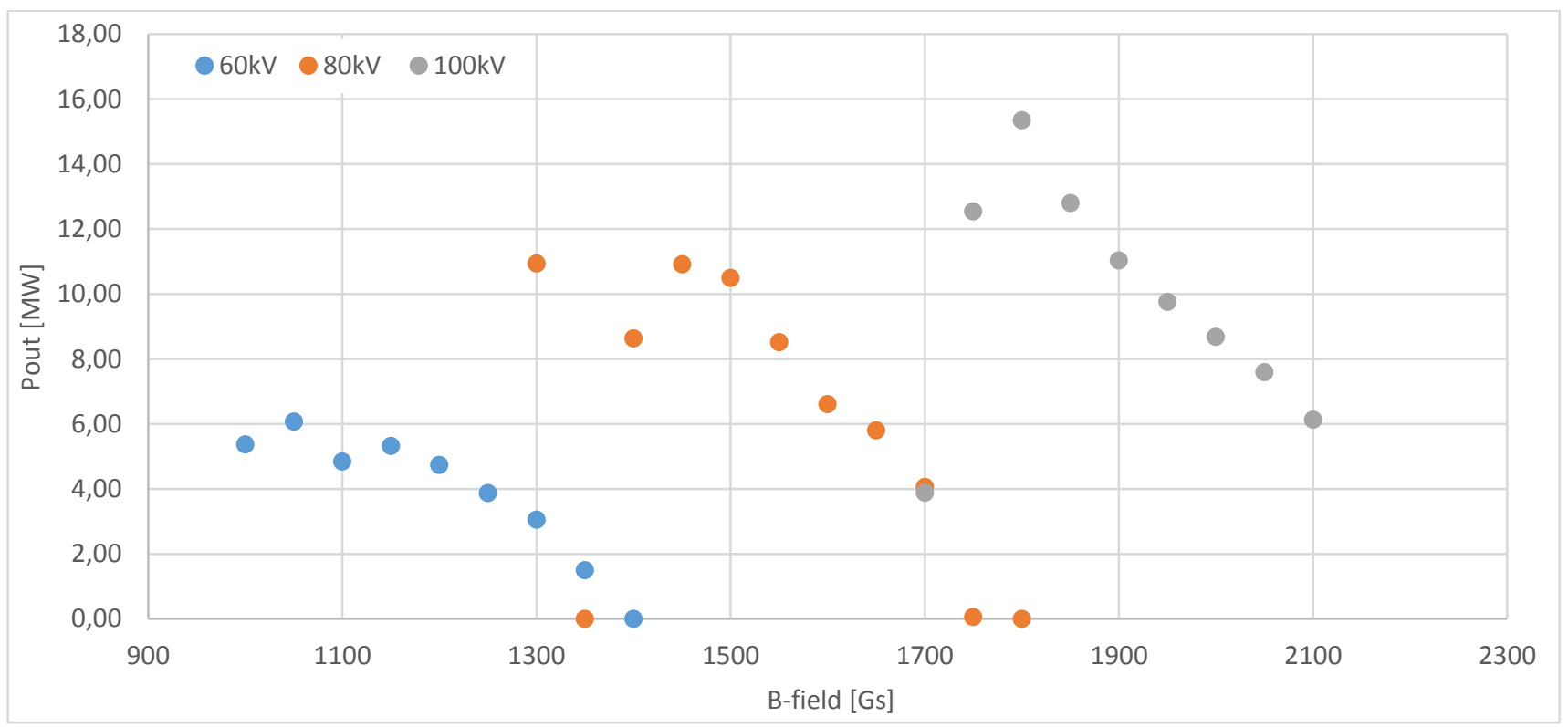

Figure 3. Output power of designed magnetron for each anode voltage as function of constant B-field

\section{EXPERIMENTAL RESULTS}

\subsection{Experimental model}

After obtaining satisfactory results of electromagnetic simulations, in which the analyzed model of a tube worked in a wide range of parameters, and the signal spectrum did not show strong parasitic modes, it was decided to make a magnetron model for experimental measurements. The anode block, cathode with a heater and power output were all made using the Company's production facilities. Having the physical model ready, the verification of the characteristics of the resonant structure dispersion matrix was possible to carry out. After assembling all components of the microwave tube and creating a vacuum inside it, dynamic magnetron measurements were made at a high power level, under real conditions. In order to carry out the measurements, a test stand with a high-voltage transformer and a modulator block powered by supercapacitors was designed and made, which, being an energy storage, aims to provide the supply voltage of the appropriate levels. The power supply with a modulator consists of a high voltage capacitor charging system, a modulator based on high voltage IGBT keys and a control system. The tube is connected by with the matched load and coupling elements using a rectangular waveguide. The pulsed power energy is absorbed in the waveguide dummy load [6]. During 
the testing of the dynamic parameters of the magnetron, stable operation was observed in the available voltage range of the high-voltage power supply (Figure 4.).

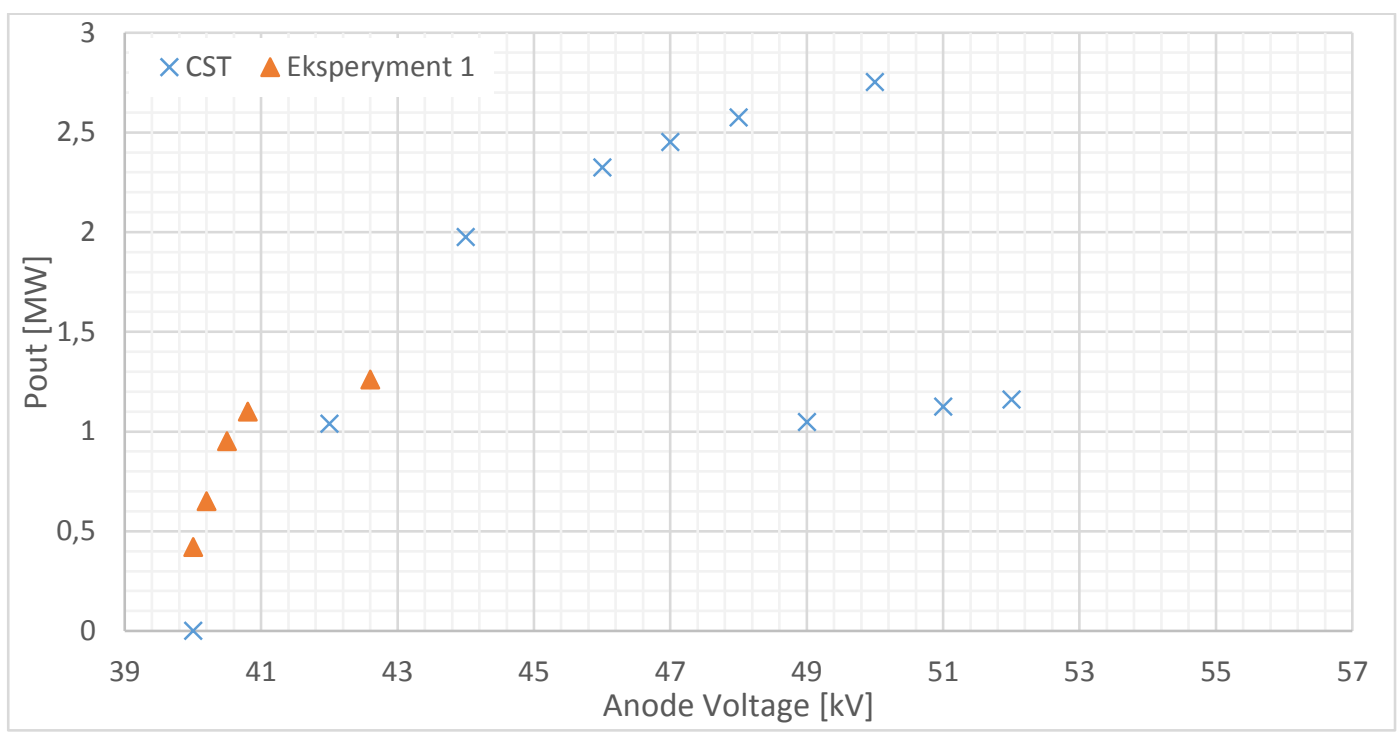

Figure 4. Comparison of simulations results and experimental results for the first version of vacuum tube and power supply.

During measurements, the maximum anode voltage of $43 \mathrm{kV}$ was reached. The final parameters of the tube differed from those analyzed during the simulation due to the limited capacity of the power source. It was necessary to find a solution to increase the power parameters of the power supply.

\subsection{Modification of power supply}

Despite the fact that the first measurements of the magnetron gave promising results, the pulse power generated by the magnetron was still far from designed. Magnetron only achieved about $1 \mathrm{MW}$ of power, where the design assumption was $5 \mathrm{MW}$. The main factor limiting the output power of the device was the power supply system, not adapted to work with such high powers. Due to too long rise time of the power signal, it was impossible to achieve the desired current value and this caused instability in the operation of the magnetron. The power supply system required a thorough redesign due to saturation of the HV transformer core. To achieve that, the power supply modulator was modified by changing the way of HV transformer keying. As a result of the modification, the operating mode was obtained, where through the primary winding of the HV transformer, in the HV capacitor charging cycle, the current magnetizing the core flows after the high voltage pulse supply cycle supplying the magnetron. The change allowed to shorten the rise time of the power signal from about $10 \mu \mathrm{s}$ to about $2.5 \mu \mathrm{s}$ and provided an overall increase in the efficiency of the magnetron power supply. Modification of modulator keying method enabled the generation of pulses with higher voltages and faster rise time. However, the tube requires seasoning for stable operation. It is a process involving increasing the resistance to voltage breakdowns and discharges that may occur in the area of interaction between the cathode and anode. Currently it was possible to make measurements for magnetic field values from $100 \mathrm{mT}$ to $140 \mathrm{mT}$ in the range up to $65 \mathrm{kV}$.

Magnetron has a wide range of operating parameters at which oscillations are excited. To achieve the designed output power and maximum efficiency, the operating point should be above the line $U(B H)$ for the $\pi$ mode. Figure 5 shows the theoretical characteristics of the threshold voltage for the designed magnetron. The dotted line is the Hull parabola. The continuous voltage $U(B H) 3 / 3 / 6$ according to the Buneman-Hartree criterion for basic type $\pi$ oscillations is marked with a solid line. Oscillations of this type are defined as type $n=3$ with three electron spokes. The lines $U(B H) 2 / 2 / 6$ and $U(B H) 4 / 2 / 6$ represent the threshold voltages for type $n=2$ (type $\pi-1$ ). In this case, the electrons form 2 and 4 spokes, respectively, which is associated with the propagation of two types of spatial harmonics when working with the nearest parasitic frequency. In the figure, the voltage working areas of the magnetron for various magnetic field values determined during experimental measurements was marked with vertical ellipsoids. 


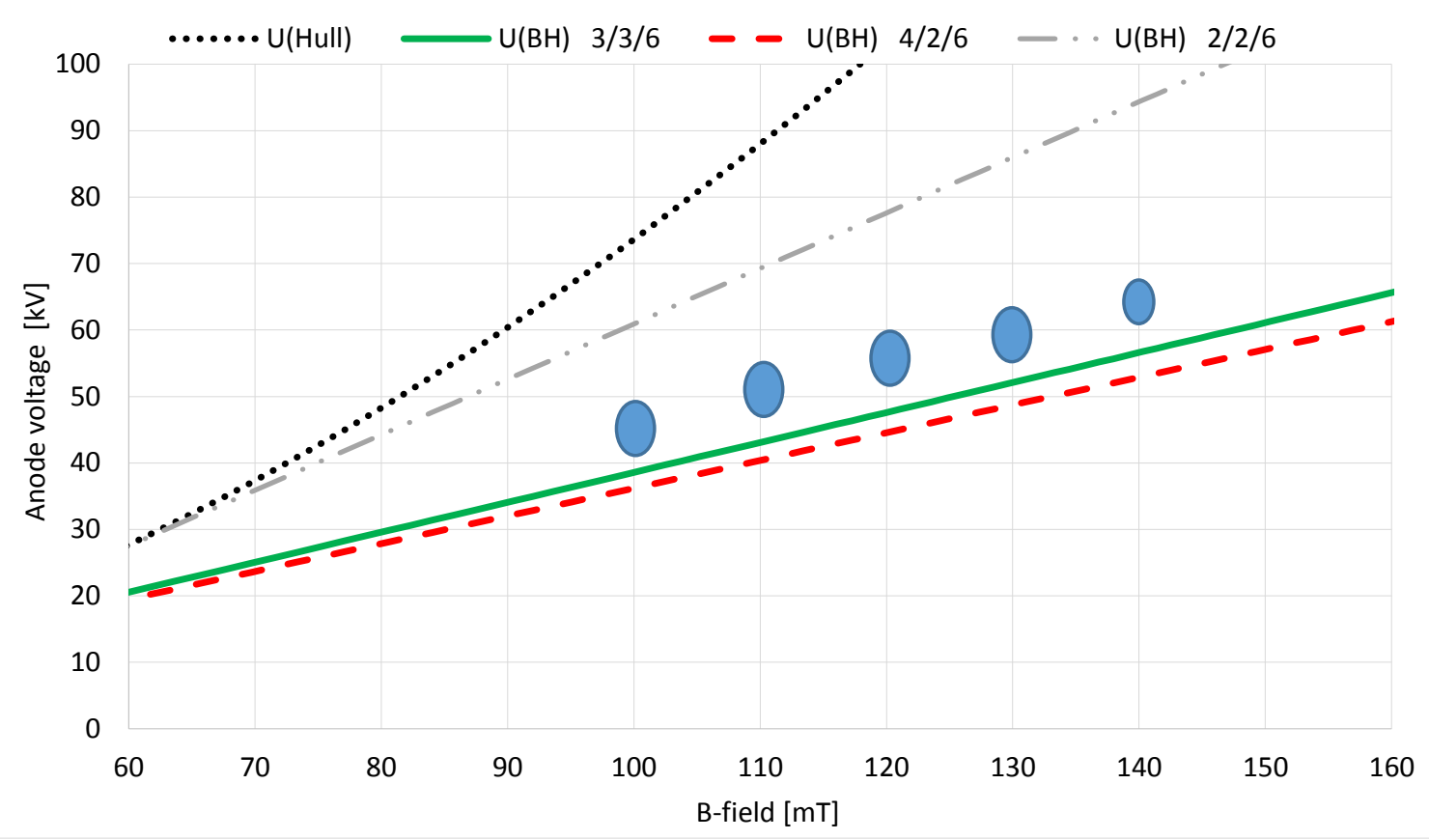

Figure 5. Areas of stable operating in measurements using new power supply. Efficiency was up to $50 \%$ for $B=130 \mathrm{mT}$

The results of the experiment agree with the theoretical linear characteristics of the anode voltage depending on the magnetic field. In practice, this relationship makes it possible to determine the operating point of magnetron by selecting the appropriate magnetic field values for the desired range of output power. Figure 6 shows the results of output power calculations obtained in the CST software for magnetic field induction $B=100 \mathrm{mT}$ and the results of experimental measurements with the old (1) and new (2) power supply. The measurement results obtained in Experiment 2 indicate that the power of the generated signal in the case of a modified power supply is obtained at a higher voltage than results from numerical simulations. At this stage, the reason for this discrepancy cannot be determined, the more so because the previous results obtained in Experiment 1 were consistent with the simulation results.

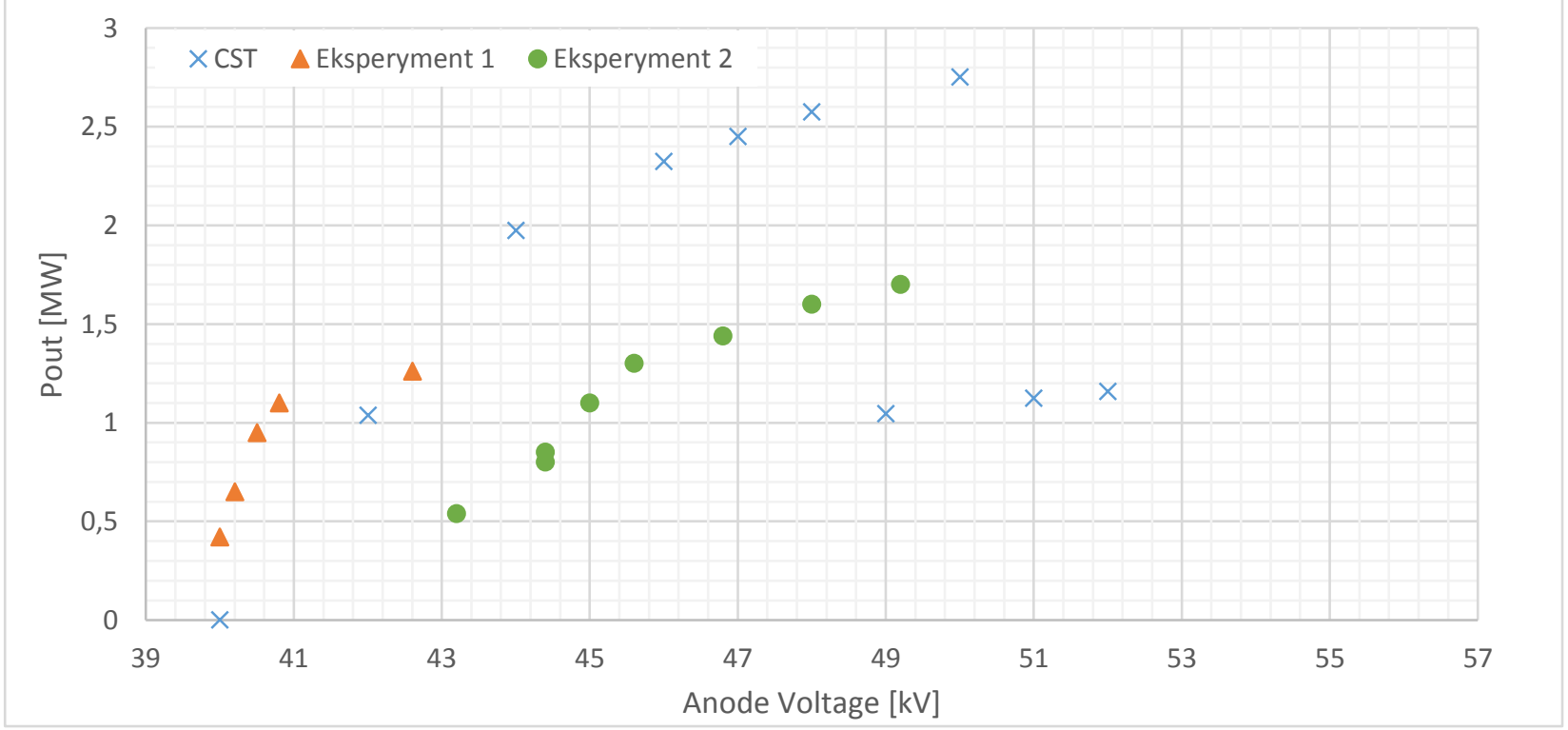

Figure 6. Comparison of output power for $B=100 \mathrm{mT}$ in CST Studio and experimental results using old(1) and new (2) power supply 


\subsection{Operating Characteristics}

Operating characteristics also called input characteristics show the dependence of the anode voltage (y axis) as a function of the anode current (x axis) at fixed magnetic induction values as a parameter. Figure 7 shows the characteristics obtained during the experimental measurements of the magnetron. Ideally, the characteristics should be straight lines inclined at a small angle to the $\mathrm{x}$ axis [10]. Figure 8 shows the input characteristics with marked waveforms of constant output power. In general, the waveforms should have a hyperbolic shape. Accurate determination of the characteristics is burdened with some inaccuracy due to the sum of errors of reading individual values - anode voltage, anode current, magnetic field and output power. In the case of results obtained during the experiment, too few measurement points were made to minimize the reading errors of the measured values.

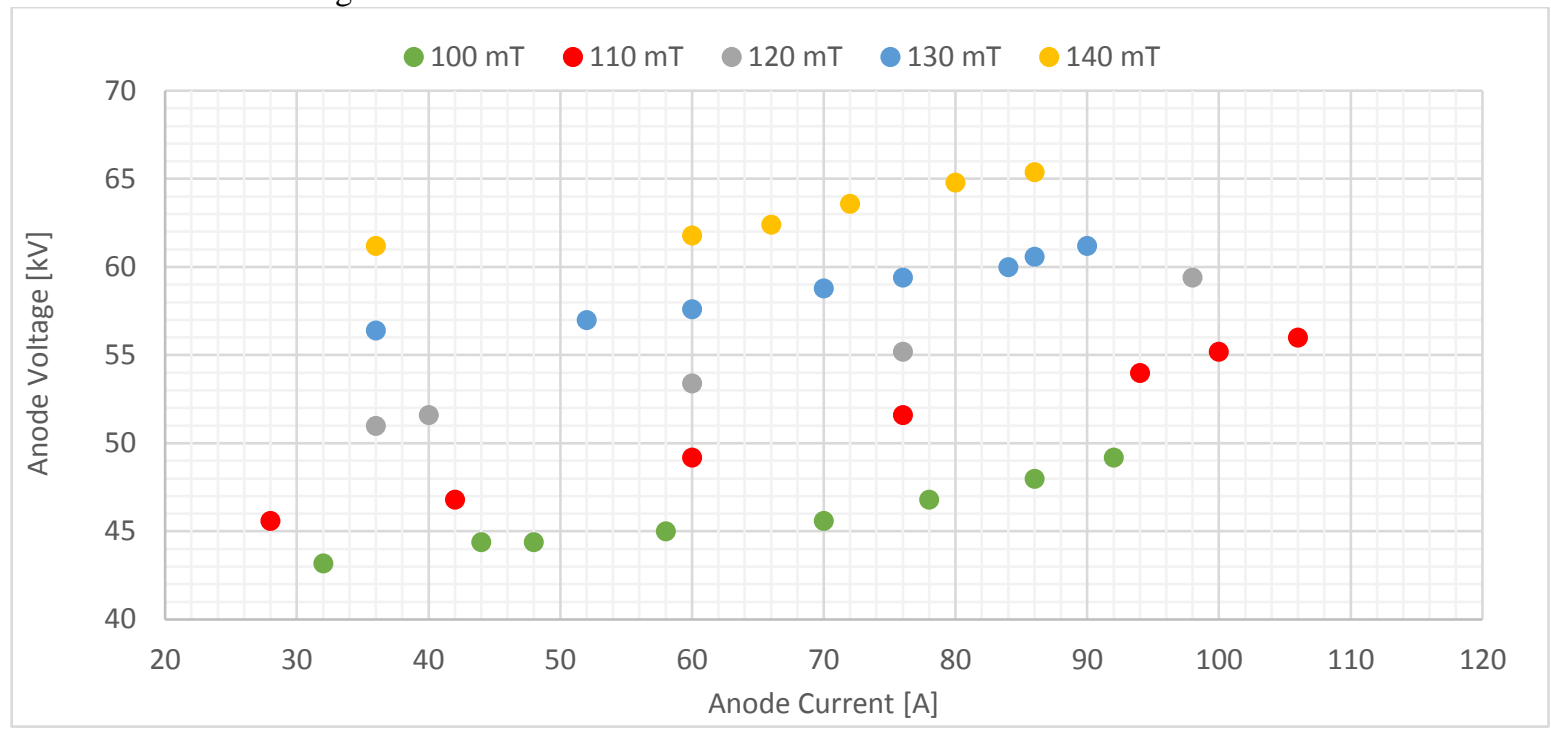

Figure 7. Voltage-current characteristics (input) of magnetron from various B-field as parameter

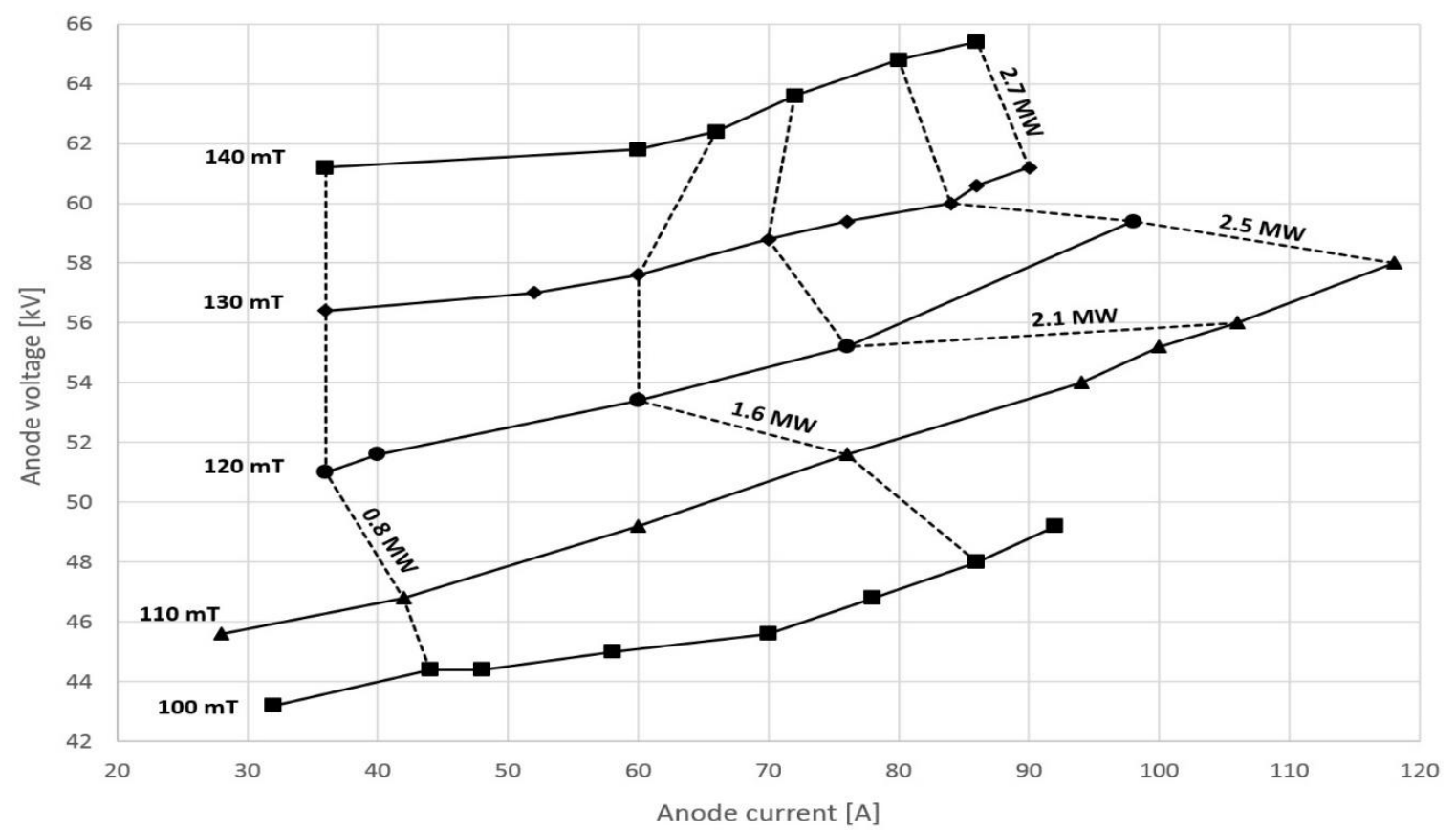

Figure 8. Operating characteristics of experimental results of magnetron. Dotted lines are lines of constant output power 
Figure 9 presents the comparison of output power as function of anode voltage for results from simulations and experiments. Both the results of the experiment and the simulation are presented for different magnetic field values.

Increasing the magnetic field allows to raise the voltage and thus obtain higher power of generated oscillations. By comparing the results significant divergencies between the RF power values obtained in the experiment and simulations can be seen. The differences can amount to about $1 \mathrm{MW}$ for all plots. The most likely explanation for such significant variances between simulations and experimental values seems to be the coexistence of three separate reasons. The first is the use of an inadequate model of electron emission from the cathode for the considered parameters of magnetron operation used in numerical simulations. The second reason may be related to the limitation of the available anode current due to saturation of the high voltage transformer core which results in the lowered RF power. The last one may be a result of a sum of random and systematic error made during power measurement. Currently the cause of the discrepancy is unknown and requires further and thorough investigation. It should be stated that the magnetron computer model considering ideal working conditions will never be able to perfectly reflect the work of a real device. Nevertheless simulation results more convergent with real device measurements are expected to obtained.

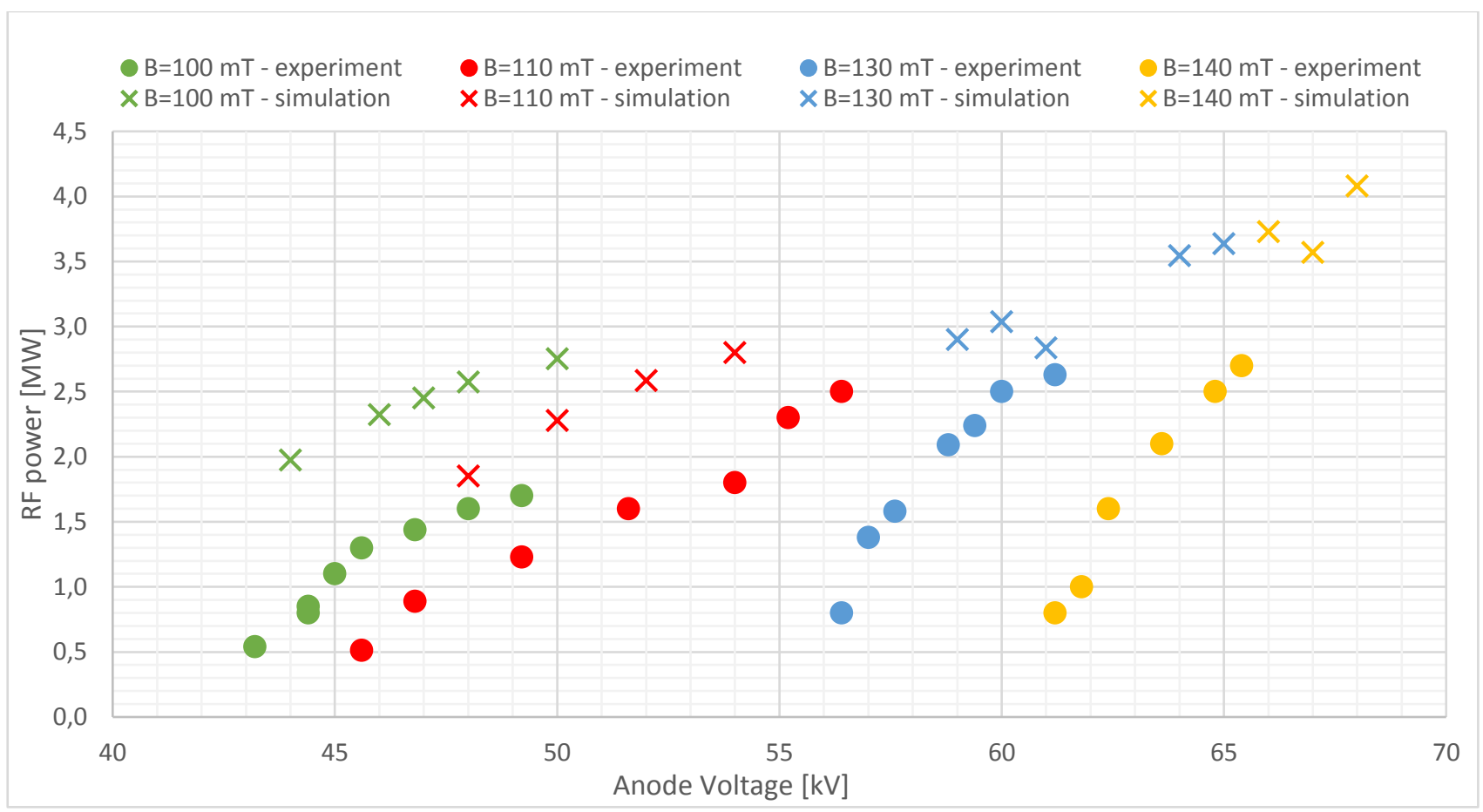

Figure 9. Comparison of output power as function of anode voltage for results from simulations and experiments.

\section{CONCLUSIONS}

Experimental measurements have shown that the design of a suitable power supply with optimal electrical parameters to power the magnetron has a significant impact on the tube's operation. Supplying the magnetron with the right voltage, made it possible to obtain results similar to those from numerical simulations and theoretical calculations. Experimental measurements have largely confirmed the correct approach to the magnetron design process. From complex analytical calculations, through numerical calculations of the resonance structure and dynamic tube operation to the production of an experimental device and testing its operation in laboratory conditions.

One of the most important results of the experiment was confirmation of the theoretical linear dependence of the anode voltage on the magnetic field, because in practice this relationship makes it possible to determine the tube operating point by selecting the appropriate magnetic field for the desired range of output power. The voltage operating area of the magnetron for various magnetic field values found during experimental measurements was correlated with high accuracy to the theoretical characteristics of the threshold voltage at which the excitation of the basic $\pi$ mode occurs.

The differences between the results of generated power obtained in the experiment and simulations can have several reasons. First of all, it should be taken into account that the magnetron is a device with non-linear dynamic characteristics, 
which induces oscillations from the chaotic movement of electrons in the area of interaction. Our computer model was not able to perfectly reproduce of real magnetic field distributions, so a homogeneous field was used in the entire interaction area. The next reason may be related to the limitation of the available anode current due to saturation of the high voltage transformer core which results in the lowered RF power. The last one factor affecting differences in results are uncertainty errors during experimental measurements. For technical reasons, the tube was seasoned to a voltage of just over $60 \mathrm{kV}$. This value is insufficient to provide the durability parameters necessary to measure the magnetron in operating conditions with nominal power, which was assumed in the early design stage. Currently it was possible to make measurements for magnetic field values from $100 \mathrm{mT}$ to $140 \mathrm{mT}$ in the voltage range up to $65 \mathrm{kV}$.

Considering the differences between simulations and experimental measurements, the obtained results confirm the effectiveness of the design methods used. Based on the experience gained, using the analogous path to other types of magnetrons, equally good results can be achieved.

\section{REFERENCES}

[1] Barker R. J. et al., [Moderm microwave and milimeter-wave power electronics], IEEE (2005).

[2] Benford J., Swegle, J.A., Schamiloglu E., [High Power Microwaves], Third Edition, Taylor \& Francis Group, New York, (2016).

[3] Birdsall C.K., Langdon A.B. [Plasma Physics via Computer Simulation], Taylor \& Francis Group, New York, 2005.

[4] Collins G. B., [Microwave Magnetrons], McGraw-Hill, (1948).

[5] Gilmour A. S., Jr., [Klystrons, Traveling wave tubes, Magnetrons, Crossed-field Amplifiers, and Gyrotrons], Aetech House, (2011).

[6] Ginzton E.L., [Microwave Measurements], McGraw-Hill Book Company, New York, (1957).

[7] Д. Е. Самсонов, [Основы расчёта и конструирования много-резонаторных магнетронов], СОВЕТСКОЕ РАДИО, Москва (1966).

[8] Szkop E., Rychlewski M., Baczewski D., Woźniak M., Różycki A., Majewski P., Błażejewicz M., Laskowski D., Weryfikacja wyników symulacji numerycznych magnetronu impulsowego dużej mocy na pasmo $L w$ warunkach doświadczalnych, KNTWRE (2018).

[9] Rychlewski M., Szkop E., Błażejewicz M., Baczewski D., Różycki A., Karpińska M., Symulacje elektromagnetyczne $z$ tadunkiem przestrzennym. Porównanie $z$ wynikami pomiarów na przykładzie magnetronów projektowanych w Kubara Lamina S.A. Elektronika: konstrukcje, technologie, zastosowania, vol $59 \mathrm{nr}$ 5, pp21-23, (2018).

[10] Wolnik J., [Magnetrony], WNT, Warszawa, (1969).

[11] CST Studio Suite 2016, https://www.cst.com/ 